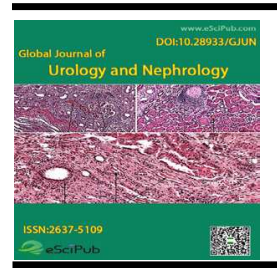

Global Journal of Urology and Nephrology

(ISSN:2637-5109)

\title{
Accuracy of Prostate Specific Antigen Density in Predicting Prostate Cancer-A single-centre UK experience
}

\author{
Rahul Pandya", Ayesha Butt", Andrei Bancu, S I Miakhil
}

Urology, North West Anglia NHS trust $\quad$ "Co-lead author ABSTRACT

Introduction and objectives: PSA has been a useful tool in identifying prostate cancer since its discovery. However relying on this alone can lead to unnecessary over investigation as it can be falsely raised in other conditions. PSA density is a possible enhanced marker for prostate biopsy indication, however it is not standard practice or recommended in national guidelines. Previous studies have shown that PSA density is most useful when the PSA is in the range of 4-10. We aim to study the effectiveness of PSA density at detecting prostate cancer within that range as well as outside that range. Methods: Retrospective single centre study at North West Anglia NHS Trust including 500 patients who had a MRI prior to prostate biopsy between July 2017 to July 2018. Patients undergoing repeat biopsy already on a cancer pathway were excluded. PSA density was calculated by dividing PSA from the prostate volume recorded on the MRI. A cut of value of PSA density was chosen at 0.15 and 0.10 . Results: Data from 500 patients with a mean age of 65 and mean PSA of 10.7 (PSA range 0.32 to 99$) .251$ (50.2\%) patients had cancer on histology with 152 having clinically significant cancer. Mean PSA density was $0.11 \mathrm{ng} / \mathrm{ml} / \mathrm{cm} 3$ with benign histology, $0.36 \mathrm{ng} / \mathrm{ml} / \mathrm{cm} 3$ for all cancer and $0.48 \mathrm{ng} / \mathrm{ml} / \mathrm{cm} 3$ for clinically significant cancer. Overall the mean PSA density in prostate cancer was found to be 0.15 . Sensitivity of PSAD at detecting all prostate cancer at cutoff of 0.10 with PSA $4-10$ was $76 \%$ and $88 \%$ for clinically significant prostate cancer (Gleason $\geq 7$ ). The negative predictive value was $94 \%$ for clinically significant prostate cancer. With PSA of 10 and PSA density of 0.10 the sensitivity was $93 \%$ (all cancer) and $96.6 \%$ (Gleason $>7$ ) with a negative predictive value of $93.7 \%$ for clin ically significant prostate cancer. At a cut off PSA density of 0.15 sensitivity and negative predictive value was $39 \%$ and $65 \%$ (all prostate cancer) and $50 \%$ and $87 \%$ (Gleason $\geq 7$ ) respectively with

PSA of 4-10. ROC curves were created for each PSA range comparing cancer cases versus benign to determine area under curve values. No statistically significant difference was found for PSA range $10 \mathrm{ng} / \mathrm{ml}$ for all cancer vs benign. However, there was a statistically significant difference when comparing AUC for PSAD in the PSA range of 4-10ng/ $\mathrm{ml}$ for all cancer vs benign. $(p<0.01)$. Conclusions: This study is useful as a high powered study with a large study population which shows a direct correlation of PSAD to Gleason scoring. PSAD cut off value of $0.10 \mathrm{ng} / \mathrm{ml} / \mathrm{cm} 3$ has a higher sensitivity and negative predictive value especially when compared against clinically significant prostate cancer. When the PSAD cut off value is increased, sensitivity decreases, however specificity improves. PSAD can be used to avoid unnecessary biopsies however also runs the risk of missing clinically significant cancers.

*Correspondence to Author: Ayesha Butt

Urology, North West Anglia NHS trust

How to cite this article:

Rahul Pandya, Ayesha Butt, Andrei Bancu, S I Miakhil. Accuracy of Prostate Specific Antigen Density in Predicting Prostate Cancer- A single-centre UK experience. Global Journal of Urology and Nephrology, 2020, $3: 23$

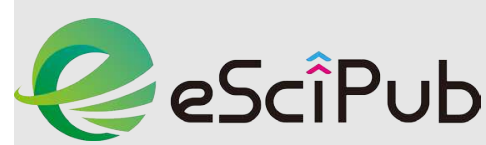
eSciPub LLC, Houston, TX USA. Website: https://escipub.com/ 


\section{Introduction}

Prostate cancer is the second most prevalent type of cancer in men and is the fifth-leading cause of cancer-related deaths worldwide 1. Serum prostatic-specific antigen (PSA) has been the standard biomarker for many years in prostate cancer diagnosis and clinical management ${ }^{2}$. PSA is a single chain glycoprotein an amino acid produced by both cancerous and non-cancerous tissue in the prostate and thus is an organ specific marker. Historically a level of $4.0 \mathrm{ng} / \mathrm{ml}$ or above was used to justify need for biopsy to screen for prostate cancer. However PSA values can vary according to age and is thought to be higher in older men due to larger size of prostatic tissue.

The most important risk factors for prostate cancer are age, ethnicity and genetics as well as a possible dietary association. Prostate cancer is rarely diagnosed in men under 40 years old, however incidence rises rapidly thereafter with a peak incidence between 65-74. Prostate cancer may manifest following routine screening in asymptomatic individuals or in more aggressive cases following metastases of prostate cancer. It is believed that without screening many cases of prostate cancer would be undiagnosed and not have any clinical significance as men are more likely to die of another unrelated cause due to the slow progression of the disease.

Symptoms associated with prostate cancer are often non-specific and may include haematuria and/or other urinary symptoms which may also be present in infection. However other times, prostate cancer may present with constitutional symptoms such as weight loss, weakness, bone pain or pathological fractures. Signs of prostate cancer will include abnormal digital rectal examination such as nodules or indurations and an elevated PSA. These clinical features would justify further evaluation for assessment of prostate cancer.

Serum PSA reflects the amount of glandular epithelium and thus the size of the prostate. As prostate gland size increases with age, you would expect the PSA concentration to increase. There is therefore no set normal range of PSA values as this can vary according to age and ethnicity. However a PSA value of $>4 \mathrm{ng} / \mathrm{ml}$ has been the most widely accepted standard used to indicate cases which would warrant an urgent urology referral for suspected prostate cancer. It is of note however that PSA may be elevated in non-malignant cases such as $\mathrm{BPH}$, prostatitis, LUTS and conversely may also be normal in prostate cancer.

Routine PSA screening for early detection of prostate cancer is still unclear due to mixed evidence in current literature that would not point towards a definite answer ${ }^{3}$. Furthermore, PSA screening is not recommended by the UK National Screening Committee mainly because this biomarker lacks specificity due to the fact that PSA is organ, but not cancer specific. This leads to over-diagnosis and over-treatment of prostate cancer ${ }^{4,5}$.

In most clinical scenarios, patients with prostate cancer undergo risk stratification by linking PSA levels and digital rectal examination (DRE) findings at admission with the Gleason score identified on prostate biopsies and MRI findings. The latter is overvalued, being used in patient counselling for further disease management, whilst PSA and DRE have limited clinical input due to their poor specificity. Gleason scores calculated from biopsies has limits of its own, such as high discordance between scores seen on prostate biopsies and radical prostatectomy specimens 6 . Therefore, a larger number of aggressive cancer cases are being inappropriately monitored. It is believed that regardless of age larger prostate glands produce more PSA compared to smaller glands hence a relatively novel parameter, PSA density (PSAD), has been subsequently proposed in an attempt to prevent unnecessary biopsies in men. PSAD is the result of serum PSA divided by prostate volume, superiorly reflecting the volume of cancer within the gland which in turn acts as more specific indicator of prostate cancer risk ${ }^{7}$. 
The higher the PSA density, the more likely it is that $\mathrm{PCa}$ is clinically significant.

Patients being investigated for prostate cancer will undergo multi-parameteric $\mathrm{MRI}$ and/or prostate biopsies of which trans-rectal ultrasound guided prostate biopsies are the most common. However prostate biopsies can be invasive and associated with a risk of infection, bleeding and pain ${ }^{8}$.

It is envisaged that measuring PSA density can possibly enhance the predictive value in detecting cancers, however this is not currently standard practice or recommended in national guidelines. Previous studies have shown that PSA density is most useful when the PSA is within the range of $4-10 \mathrm{ng} / \mathrm{ml}$ due to diagnostic uncertainty of PSA within that range ${ }^{9}$.

\section{Methods}

This was a retrospective single centre observational study conducted at North West Anglia NHS Trust over a one year period between July 2017 and July 2018. The study included a total of 500 patients who had a multi parametric-MRI prior to prostate biopsy.

Eligible patients in this study were under investigation for prostate cancer due to a raised PSA or abnormal digital rectal examination findings. These patients were all deemed fit to undergo all the relevant procedures in the pathway including mp-MRI, trans-rectal ultrasound and prostate biopsies.

Patients were excluded if they were already on a current cancer pathway and underwent prostate biopsies for staging or active surveillance protocol. Patients were also excluded if MRI scans were contra-indicated for any reason.

PSA density was calculated by dividing total PSA over the prostate volume. The total prostate volume was measured from the multi-parametric MRI scan only.

Clinically significant cancer was defined as Gleason $\geq 7$ as taken from the histology results of the prostate biopsy.
The electronic medical records of patients in this study were examined to look at the radiological findings of the MRI, as well as the histology findings of the prostate biopsy. We also reviewed the multi-disciplinary meeting records.

Data was split in to two groups for analysis depending on the PSA ranges with one group looking at PSA values of $4-10 \mathrm{ng} / \mathrm{ml}$ and the second looking at PSA levels outside of that range.

\section{Statistical Analyses}

The data relating to histology was categorical in nature which was presented as an absolute figure.

Categorical data was analysed and compared using two by two contingency tables to present the results and compare the findings. This was used to calculate the diagnostic accuracy estimates including specificity, sensitivity, positive and negative predictive values alongside $95 \%$ confidence intervals.

Further analysis was performed with SPSS software. Receiver operating characteristic curves (ROC) were created to compare the diagnostic ability of PSA density and age related PSA for all cancer as well as clinically significant cancer in both PSA ranges.

Area under the curve (AUC) was calculated from these ROC curves and comparisons were made for both PSA and PSA density.

\section{Results}

Data was collected from 500 patients with a mean age of 65 and mean PSA of $10.7 \mathrm{ng} / \mathrm{ml}$. Of the 500 patients $251(50.2 \%)$ had cancer on histology with 152 having clinically significant prostate cancer $(30.4 \%)$. Overall the mean PSA density was $0.11 \mathrm{ng} / \mathrm{ml} / \mathrm{cm} 3$ with benign histology. $36 \mathrm{ng} / \mathrm{ml} / \mathrm{cm} 3$ for all cancer and $0.48 \mathrm{ng} / \mathrm{ml} / \mathrm{cm} 3$ for clinically significant cancer. (Table 1)

A total of $321(64.2 \%)$ patients had a PSA between $4-10 \mathrm{ng} / \mathrm{ml}$. The mean PSA density within the PSA range of $4-10 \mathrm{ng} / \mathrm{ml}$ was calculated for each Gleason score and found to 
be $0.11 \mathrm{ng} / \mathrm{ml} / \mathrm{cm} 3$ for benign, 0.13 for Gleason 6 ,

0.16 for Gleason 7, 0.18 for Gleason 8 and 0.21 for Gleason 9. The remaining 179 (35.8\%) patients had a PSA either less than $4 \mathrm{ng} / \mathrm{ml}$ $(n=43)$ or greater than $10 \mathrm{ng} / \mathrm{ml}(\mathrm{n}=136)$ which included one extreme PSA value of $1166 \mathrm{ng} / \mathrm{ml}$.
Within this group the mean PSA density was calculated for each Gleason score and found to be 0.11 for benign, 0.28 for Gleason 6, 0.54 for Gleason 7, 0.67 for Gleason 8, 0.74 for Gleason 9 and 0.56 for Gleason 10.

\section{Table 1}

\begin{tabular}{|l|l|l|l|l|l|l|}
\hline Gleason & Mean PSA density & Median PSA density & Range & SD & Average Age & Average PSA \\
\hline 0 & 0.11 & 0.1 & $0.01-0.52$ & 0.065 & 63.2 & 6.87 \\
\hline 6 & 0.17 & 0.14 & $0.03-1.18$ & 0.15 & 64.6 & 8.86 \\
\hline 7 & 0.36 & 0.23 & $0.05-2.39$ & 0.4 & 66.8 & 15.92 \\
\hline 8 & 0.93 & 0.4 & $0.09-9.04$ & 1.9 & 73.14 & 82.33 \\
\hline 8 & 0.52 & 0.36 & $0.09-1.46$ & 0.42 & 73.05 & 28.15 \\
\hline 9 & 0.56 & 0.33 & $0.11-3.62$ & 0.278 & 71.7 & 21.3 \\
\hline 10 & 0.56 & 0.56 & $0.48-0.65$ & 0.12 & 72 & 16.05 \\
\hline
\end{tabular}

Table 2

\begin{tabular}{|l|l|l|l|l|}
\hline & PSA 4-10 & & \multicolumn{3}{|l|}{ PSA $<4$ or >10 } \\
\hline PSA density cut off & Cancer & Gleason 7> & Cancer & Gleason 7> \\
\hline 0.1 & & & & \\
\hline Sensitivity & $76 \%$ & $88 \%$ & $93 \%$ & $97 \%$ \\
\hline Specificity & $58 \%$ & $51 \%$ & $63 \%$ & $50 \%$ \\
\hline PPV & $57 \%$ & $31 \%$ & $82 \%$ & $65 \%$ \\
\hline NPV & $77 \%$ & $94 \%$ & $83 \%$ & $94 \%$ \\
\hline 0.15 & & & & \\
\hline Sensitivity & $39 \%$ & $50 \%$ & $87 \%$ & $91 \%$ \\
\hline Specificity & $85 \%$ & $81 \%$ & $75 \%$ & $60 \%$ \\
\hline PPV & $65 \%$ & $40 \%$ & $86 \%$ & $69 \%$ \\
\hline NPV & $65 \%$ & $87 \%$ & $76 \%$ & $87 \%$ \\
\hline
\end{tabular}

Table 3: Predictive values for age specific PSA (PSA $<4$ or $>10 \mathrm{ng} / \mathrm{ml}$ ) for all cancer

\begin{tabular}{|l|l|l|l|l|}
\hline & Benign & Cancer & & \\
\hline PSA raised & 40 & 112 & $73.70 \%$ & PPV \\
\hline PSA not raised & 24 & 3 & $88.90 \%$ & NPV \\
\hline & 64 & 115 & & \\
\hline & $37.50 \%$ & $97.40 \%$ & & \\
\hline & Specificity & Sensitivity & & \\
\hline
\end{tabular}


Table 4: Predictive values for age specific PSA (PSA 4-10 ng/ml) for all cancer

\begin{tabular}{|l|l|l|l|l|}
\hline & Benign & Cancer & & \\
\hline PSA raised & 179 & 132 & $42.40 \%$ & PPV \\
\hline PSA not raised & 6 & 4 & $60.00 \%$ & NPV \\
\hline & & & & \\
\hline & $3.20 \%$ & $97.10 \%$ & & \\
\hline & Specificity & Sensitivity & & \\
\hline
\end{tabular}

Table 5: Area under the curve analysis PSA and PSA density in all prostate cancers versus benign cases with a PSA value lower than 4 or greater than 10.

\begin{tabular}{|l|l|l|l|l|l|}
\hline Test Result Variable(s) & Area & Std. Error & Asymptotic Sig. ${ }^{\mathrm{b}}$ & \multicolumn{2}{|l|}{ Asymptotic 95\% Confidence Interval } \\
\hline & & & & Lower Bound & Upper Bound \\
\hline PSA $(<4,>10)$ & 0.852 & 0.032 & 0 & 0.79 & 0.914 \\
\hline PSAD $(<4,>10)$ & 0.895 & 0.024 & 0 & 0.847 & 0.942 \\
\hline
\end{tabular}

Table 6: Area under the curve analysis for PSA and PSA density in all prostate cancers versus benign cases with a PSA value between 4 and 10.

\begin{tabular}{|l|l|l|l|l|l|}
\hline Test Result Variable(s) & Area & Std. Error & Asymptotic Sig. ${ }^{\mathrm{b}}$ & \multicolumn{2}{l|}{ Asymptotic 95\% Confidence Interval } \\
\hline & & & & Lower Bound & Upper Bound \\
\hline PSA $(<4,>10)$ & .557 & .033 & .082 & .493 & .621 \\
\hline PSAD $(<4,>10)$ & .700 & .030 & .000 & .642 & .758 \\
\hline
\end{tabular}

Table 7: Area under the curve analysis for PSA and PSA density in clinically significant prostate cancers versus benign cases with a PSA value between 4 and 10 .

\begin{tabular}{|l|l|l|l|l|l|}
\hline Test Result Variable(s) & Area & Std. Error & Asymptotic Sig. & \multicolumn{2}{l|}{ Asymptotic 95\% Confidence Interval } \\
\hline & & & & Lower Bound & Upper Bound \\
\hline PSA $(<4,>10)$ & .661 & .037 & .000 & .589 & .733 \\
\hline PSAD $(<4,>10)$ & .752 & .034 & .000 & .685 & .818 \\
\hline
\end{tabular}




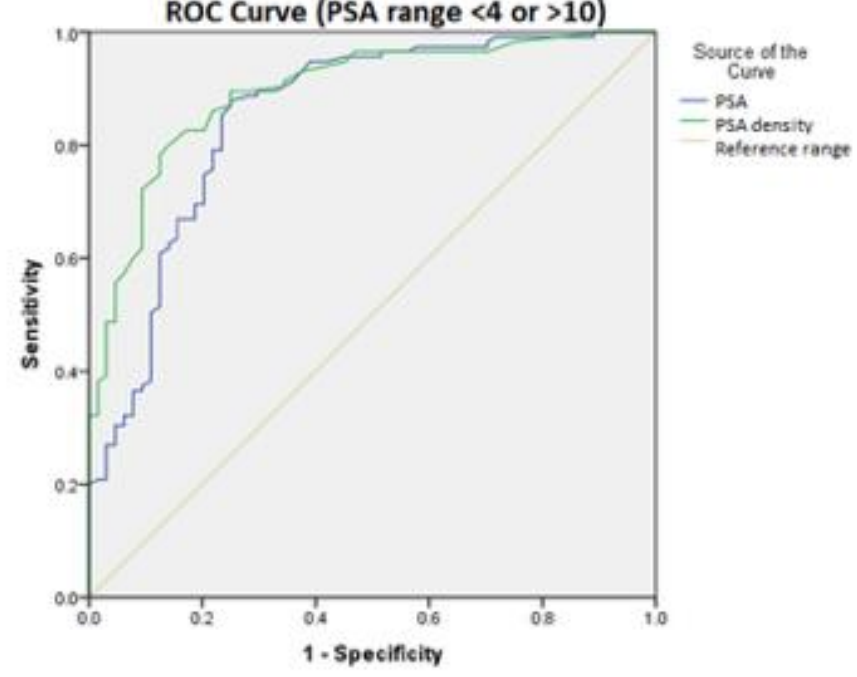

Graph 1: Joint ROC curve for PSA and PSA density in all prostate cancers versus benign cases with a PSA value lower than 4 or greater than 10.

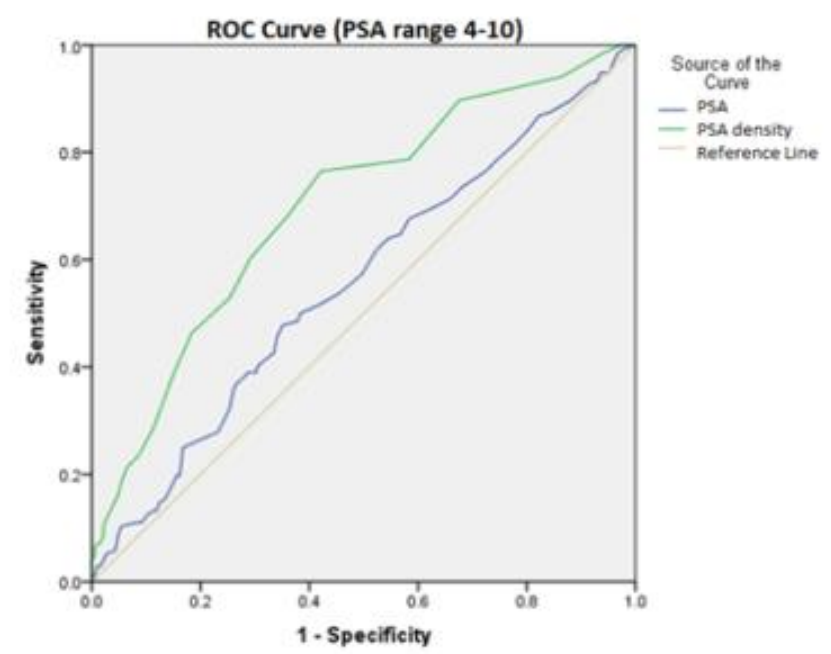

Graph 2: Joint ROC curve for PSA and PSA density in all prostate cancers versus benign cases with a PSA value between 4 and 10 .

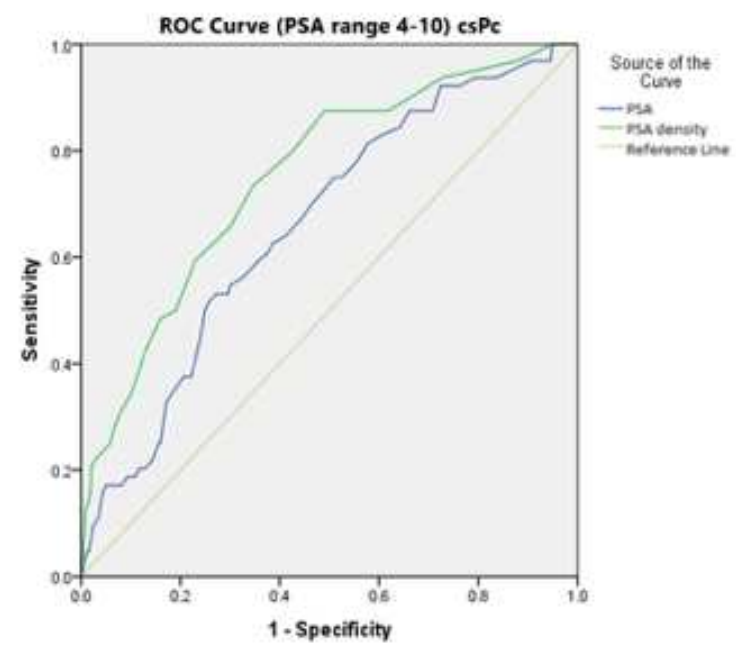

Graph 3: Joint ROC curve for PSA and PSA density in clinically significant prostate cancers versus benign cases with a PSA value between 4 and 10 
There is a statistically significant difference between the mean PSA density values of cancer positive biopsies versus benign in both of the PSA ranges. (T test $p<0.001$ ).

Two by two contingency tables were used to analyse the data and to establish diagnostic elements for both all cancer and clinically significant cancer. PSA density cut-off values were arbitrarily chosen at $0.15 \mathrm{ng} / \mathrm{ml} / \mathrm{cm} 3$ and $0.10 \mathrm{ng} / \mathrm{ml} / \mathrm{cm} 3$ (Table 2).

Sensitivity of 0.10 at detecting all prostate cancer and clinically significant cancer with PSA $4-10 \mathrm{ng} / \mathrm{ml}$ was $76 \%$ and $88 \%$ respectively. The negative predictive value was $94 \%$ for clinically significant prostate cancer. Further values have been presented in Table 2 .

With PSA of $<4$ or $>10$ and PSA density of 0.10 the sensitivity was $93 \%$ (all cancer) and $96.6 \%$ (Gleason $>7$ ) with a negative predictive value of $93.7 \%$ for clinically significant prostate cancer.

At a cut off PSA density of 0.15 sensitivity and negative predictive value was $39 \%$ and $65 \%$ (all prostate cancer) and $50 \%$ and $87 \%$ (clinically significant cancer) respectively with PSA of 4-10.

The predictive values were also calculated for raised age specific PSA to compare these values to those for PSA density. (Table 3)

It is noticeable that age related PSA is highly sensitive no matter the PSA range, however extremely non-specific in both the PSA ranges and in particular in the $4-10 \mathrm{ng} / \mathrm{ml}$ range. ROC curves were created to compare the performance of PSA and PSA density in both the PSA ranges (see Figures below). From these graphs curves, Area under the Curve (AUC) was calculated and comparison made.

When assessing the PSA range $<4$ or $>10 \mathrm{ng} / \mathrm{ml}$ for all cancer vs benign there was no statistically significant difference in AUC ( $p=0.2324)$ (Graph 1, Table 5).

However, there was a statistically significant difference when comparing AUC for PSA and $P S A D$ in the PSA range of $4-10 \mathrm{ng} / \mathrm{ml}$ for all cancer vs benign. $(p<0.01)($ Graph 2, Table 6).
The ROC curve for PSA range of $4-10 \mathrm{ng} / \mathrm{ml}$ for clinically significant cancer versus benign demonstrated a difference in area under the curve, however this was not statistically significant as it was for all cancer. ( $p$ 0.0976) (Graph 3, Table 7).

\section{Discussion/Conclusion}

Screening for prostate cancer with PSA and other derivatives such as PSAD has been proven controversial throughout the published literature, yielding mixed results in terms of benefits. It has been shown that despite reducing the overall cancer-specific mortality, PSA screening might lead to unnecessary biopsies and increased patient morbidity postsurgery and radiotherapy ${ }^{10}$. On the other hand, PSAD was viewed as a viable alternative since it better reflects the amount of cancerous tissue within the prostatic gland. Our study claims the fact that PSAD performed better than PSA in assessing patients with prostate cancer having a PSA value between 4 and 10 .

Most of the published data reported similar findings for all cancers in this particular interval $11,12,13$. However, as the Gleason score progresses, the favourable difference becomes insignificant. This might be attributed to serum PSA calculation which was pre-adjusted to age. Our results suggest that overall, PSAD performed better than PSA in diagnosing prostatic cancer, particularly in those patients with Gleason 6 disease with a serum PSA ranging from 4 to 10 . In terms of management, all these cases would fall into the low risk category. In current practice, patients are offered a choice of treatments, ranging from radical prostatectomy and radiotherapy to active surveillance. The evidence does not show a difference in the number of deaths from prostate cancer among people offered either of these options. Disease progression and distant metastasis at 10 years can be prevented by $13 \%$ and $5 \%$ respectively, if patients undergo radical treatment when compared to active surveillance. However, debilitating side-effects such as urinary incontinence and erectile dysfunction are 
more often reported following these procedures and almost all cases are opted for active surveillance. The issue with all these patients is that they have already undergone an unnecessary prostate biopsy and often complain of unpleasant side effects (haematospermia, haematuria, per rectum bleeding and pain) ${ }^{14}$.

A PSAD cut off value of $0.10 \mathrm{ng} / \mathrm{ml} / \mathrm{cm} 3$ has a higher sensitivity and negative predictive value especially when compared against clinically significant prostate cancer. When the PSAD cut off value is increased, sensitivity decreases, however specificity improves.

This study is useful as a high powered study with a large study population which shows a direct correlation of PSAD to Gleason scoring.

PSAD could thus be used to avoid unnecessary biopsies however this comes with a risk of missing clinically significant prostate cancer for example, by using a cut-off of $0.15,50 \%$ of clinically significant cancers are missed. Additionally, Lowering the cut-off to 0.10 would minimise the chances of missing a Gleason $>7$ cancer to $12 \%$.

Although PSAD has not been found to be an appropriate tool for populational prostate cancer screening, it is still useful in assessing patients based on their individual clinical status. Therefore, older or frail patients especially with co-morbidities could be spared from the burden of a prostate biopsy and cancer diagnosis whilst the young and fitter could be evaluated with a lower PSAD cut-off in order to reduce the chance of missing significant disease.

PSA density can help improve the accuracy of detecting prostate cancer but may need more evaluation as it remains partially inconclusive.

\section{References}

1. Siegel $R$, Miller $K$, Jemal $A$. Cancer statistics, 2019. CA: A Cancer Journal for Clinicians. 2019;69(1):7-34.

2. Prensner J, Rubin $M$, Wei J, Chinnaiyan A. Beyond PSA: The Next Generation of Prostate Cancer Biomarkers. Science Translational Medicine. 2012;4(127): 127rv3-127rv3.
3. Ilic D, Djulbegovic M, Jung J, Hwang E, Zhou Q, Cleves A et al. Prostate cancer screening with prostate-specific antigen (PSA) test: a systematic review and metaanalysis. BMJ. 2018;:k3519.

4. Ebell M, Thai T, Royalty K. Cancer screening recommendations: an international comparison of high income countries. Public Health Reviews. 2018;39(1).

5. Saini S. PSA and beyond: alternative prostate cancer biomarkers. Cellular Oncology. 2016;39(2):97-106.

6. Corcoran N, Hong M, Casey R, Hurtado-Coll A, Peters $\mathrm{J}$, Harewood $\mathrm{L}$ et al. Upgrade in Gleason score between prostate biopsies and pathology following radical prostatectomy significantly impacts upon the risk of biochemical recurrence. BJU International. 2011;108(8b):E202-E210.

7. Kundu S, Roehl K, Yu X, Antenor J, Suarez B, Catalona W. Prostate Specific Antigen Density Correlates With Features of Prostate Cancer Aggressiveness. Journal of Urology. 007;177(2):505-509.

8. Ahmed H, El-Shater Bosaily A, Brown L, Gabe R, Kaplan R, Parmar M et al. Diagnostic accuracy of multi-parametric MRI and TRUS biopsy in prostate cancer (PROMIS): a paired validating onfirmatory study. The Lancet. 2017;389(10071):815-822.

9. Luderer A, Chen Y, Soriano T, Kramp W, Carlson $G$, Cuny $C$ et al. Measurement of the proportion of free to total prostate-specific antigen improves diagnostic performance of rostate-specific antigen in the diagnostic gray zone of total prostatespecific antigen. Urology. 1995;46(2):187-194.

10. Tikkinen KAO, Dahm P, Lytvyn L, et al. Prostate cancer screening with prostate-specific antigen (PSA) test: a clinical practice guideline. BMJ 2018:362:k3581

11. Jue JS, Barboza MP, Prakash NS, Venkatramani V, Sinha VR, Pavan N, et al. Re-examining Prostate-specific Antigen (PSA) density: defining the optimal psa range and patients for using psa density to predict prostate cancer using extended template biopsy. Urology. 2017;105:123-8

12. Elliott CS, Shinghal R \& Presti JC, Jr. (2008) The performance of prostate specific antigen, rostate specific antigen density and transition zone density in the era of extended biopsy schemes. $J$ Urol 179, 1756-1761.

13. Nordstrom T, Akre O, Aly M, Gronberg H, Eklund M. Prostate-specific antigen (PSA) density in the diagnostic algorithm of prostate cancer. Prostate Cancer Prostatic Dis. 2017;21(1):57-63.

14. National Institute for Health and Care Excellence (2019) Prostate cancer: diagnosis and 
Rahul Pandya et al., GJUN, 2020 3:23

management (NICE Guideline 131). Available at:

https://www.nice.org.uk/

guidance/ng131/evidence/january-2014-full-

guideline-pdf-6781033550 [Accessed 1509 2019]. 